\title{
Laparoscopic Management of Gastric Torsion After Sleeve Gastrectomy
}

\author{
Christian Hernandez Murcia, MD, Pedro Garcia Quintero, MD, \\ Jorge Rabaza, MD, FACS, FASMBS, Anthony Gonzalez, MD, FACS, FASMBS \\ Department of General and Bariatric Surgery, Baptist Health South Florida, Miami, FL, USA (all authors).
}

\begin{abstract}
Introduction: Gastric volvulus occurs primarily when the stomach suffers torsion on itself due to laxity, elongation, or agenesis of the stomach ligamentous attachments or secondary to diaphragmatic hernias. Gastric torsion after sleeve gastrectomy is a rare complication. We present a case report of 3 patients with gastric torsion after sleeve gastrectomy.

Case Description/Technique Description: Three patients with gastric torsion after sleeve gastrectomy were identified. Time between sleeve gastrectomy and gastric torsion symptomatology was 25, 211, and 98 days. Endoscopy established the diagnosis in all patients. Operative findings were organoaxial torsion due to adhesions of the sleeve's staple line to the liver in all cases. Gastropexy was required in 1 case after laparoscopic lysis of adhesions. Recovery was uneventful for all patients, without torsion recurrence.

Discussion: Because sleeve gastrectomy alters the normal stomach attachments, gastric torsion must be considered after sleeve gastrectomy in patients with any degree of obstruction symptoms, at any time of the postoperative course. Endoscopy is a valuable tool for the diagnosis. Laparoscopic torsion reduction by lysis of adhesions is successful, with or without gastropexy.
\end{abstract}

Key Words: Gastric volvulus, Gastric torsion, Sleeve gastrectomy, Lysis of adhesions, Gastropexy.

\footnotetext{
Citation Murcia CH, Quintero PG, Rabaza J, Gonzalez A. Laparoscopic management of gastric torsion after sleeve gastrectomy. CRSLS e2014.00143. DOI: 10.4293/CRSLS.2014.00143.

Copyright (c) 2015 by SLS, Society of Laparoendoscopic Surgeons. This is an open-access article distributed under the terms of the Creative Commons Attribution-Noncommercial-ShareAlike 3.0 Unported license, which permits unrestricted noncommercial use, distribution, and reproduction in any medium, provided the original author and source are credited.

Drs. Gonzalez and Rabaza have relationships with Intuitive Surgical as teachers, speakers, and proctors.

Address correspondence to: Anthony Gonzalez MD, FACS, FASMBS, Department of General and Bariatric Surgery, Baptist Health South Florida, 7800 SW 87 Avenue, Suite B210, Miami, FL 33173. Telephone: (305) 271-9777, Fax: (305) 598-7711, E-mail: anthonyg@baptisthealth.net
}

\section{INTRODUCTION}

The stomach is normally positioned and fixed by ligamentous attachments to the spleen, liver, and diaphragm. Gastric volvulus (GV) occurs primarily when the stomach suffers torsion on itself due to the laxity or elongation of these attachments and secondary to fixation at a specific point such as adhesions, tumors, or diaphragmatic and hiatal hernias. GV may be mesenteroaxial (when the stomach suffers torsion on its short axis), organoaxial (when the stomach suffers torsion along its long axis), and mixed (mesenteroaxial and organoaxial). ${ }^{1}$ Positioned between gastric bypass and adjustable gastric banding due to its safety and good results, sleeve gastrectomy (SG) is well established as a treatment for morbid obesity. ${ }^{2}$ The most common complica- tions related to SG are leaks (0.7\%), abscesses (0.7\%), hemorrhages $(0.7 \%)$, and strictures $(0.7 \%){ }^{3}$ Furthermore, its mortality rate $(0.5 \%)$ is quite low. ${ }^{4} \mathrm{SG}$ popularity worldwide has grown significantly among bariatric/metabolic procedures and now encompasses $27.8 \%$ of procedures performed in the past 8 years. ${ }^{5}$ Due to this rapid growth, new complications are sure to be noticed.

Once SG has been performed, GV is no longer a valid term to be used when the remaining stomach "twists, folds, or turns," because the attachments have been disrupted and make the sleeve susceptible to this problem. Therefore, we believe that gastric torsion (GT) is a better term after SG or any gastric procedure that detaches the stomach from its natural points of fixation. GT after SG is a rare complication. To our knowledge, there are only 2 
Laparoscopic Management of Gastric Torsion After Sleeve Gastrectomy, Hernandez Murcia et al.

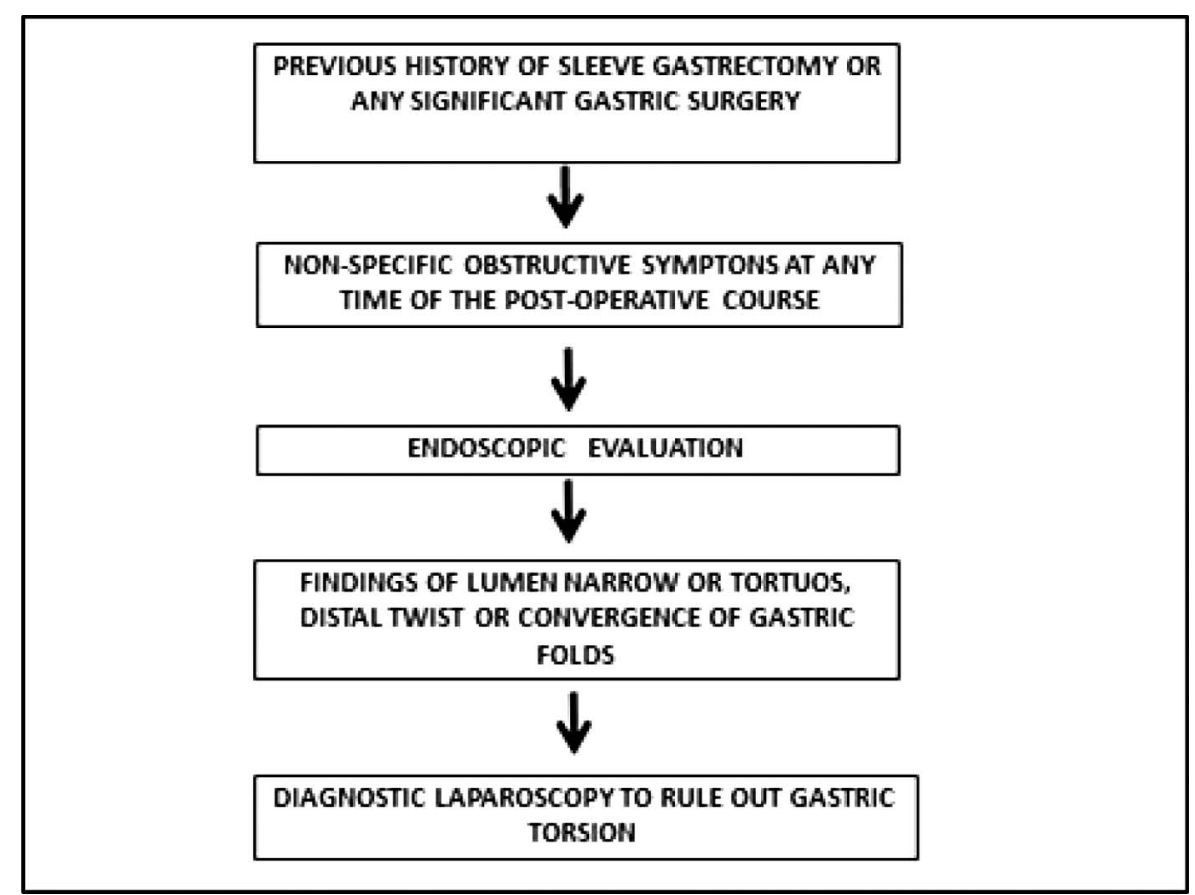

Figure 1. Algorithm for the diagnosis of gastric torsion.

cases $^{6,7}$ reported in the literature. Here, we expand on these reports by presenting 3 patients diagnosed with GT after SG and their subsequent management.

\section{Patient 1}

A 38-year-old woman, with a body mass index (BMI) of 46 $\mathrm{kg} / \mathrm{m}^{2}$, whose comorbidities included diabetes mellitus (DM) type 2, hypertension, sleep apnea, hypothyroidism, and asthma, underwent SG. She presented with swallowing difficulties, reflux, abdominal pain, and diarrhea 25 days postoperatively. An upper endoscopy revealed the lumen of the SG to be narrow and slightly tortuous, without any obvious obstruction. Plans for laparoscopy were made after failed medical therapy, which included dietary changes, antiemetics medications, and high-dose proton pump inhibitors over a week's time. Operative findings were organoaxial GT, secondary to adhesions between the mid portion of the sleeve's staple line and the liver. The adhesions were lysed carefully in this area. An intraoperative endoscopy was performed and revealed resolution of the torsion of the sleeve. Her recovery was uneventful.

Eleven months later, she returned complaining of nausea and vomiting, and endoscopy found the sleeve to be normal. A hiatal hernia type 1 , not previously noted, was found at this time. She was instructed regarding diet and behavioral modification.
Fifteen months after her primary SG, she was converted to gastric bypass, due to weight loss failure with a BMI of 40.44 $\mathrm{kg} / \mathrm{ms}^{2}$. The symptomology related to the GT never recurred.

\section{Patient 2}

A 68-year-old woman, with initial BMI of $43 \mathrm{~kg} / \mathrm{ms}^{2}$ and comorbidities including DM type 2, hypertension, dyslipidemia, and gastroesophageal reflux disease, underwent SG and repair of a small crural defect. Her recovery was uneventful and by 5 months after SG her DM was resolved.

She subsequently presented with dysphagia 211 days after the SG. Endoscopy findings demonstrated that the SG had no stricture, but a twist of the distal sleeve was noted that was nonobstructive. The decision was made to proceed to the operating room due to these findings. During diagnostic laparoscopy, the operative findings included organoaxial GT secondary to adhesions between the sleeve's staple line and the liver, falciform ligament, and retroperitoneum (Fig. 1). A laparoscopic lysis of adhesion was performed; the stomach was untwisted and clockwise rotated and returned to the normal anatomic position. Multiple attempts were made to fixate the sleeve to the greater omentum and gastrocolic ligament, but they were not successful in maintaining correct sleeve orientation, due to its tendency to 
retwist. Therefore, gastropexy to the retroperitoneum was performed with absorbable 2-0 Vicryl (Ethicon Inc, Somerville, NJ), interrupted sutures.

At 1-year follow-up after the primary SG, her BMI was 32.7 $\mathrm{kg} / \mathrm{m}^{2}$, her comorbidities were resolved, and she had no obstructive symptoms.

\section{Patient 3}

A 50 -year-old woman with an initial BMI of $35.27 \mathrm{~kg} / \mathrm{m}^{2}$ and DM treated with combined medications underwent a SG. Though her initial recovery was uneventful, she presented with intractable nausea and vomiting 98 days after surgery.

An endoscopy demonstrated a hiatal hernia and convergence of folds in a spiral appearance in the mid portion of the stomach. No obstruction was noted with the endoscope.

Due to the spiral appearance of the folds, plans were made for laparoscopic lysis of adhesions, derotation of GT, and concomitant hiatal hernia repair. Operative findings were organoaxial GT secondary to adhesions between the sleeve's staple line and the liver. A robotic lysis of adhesions was performed. Attempts were made to fixate the sleeve to the gastrocolic ligament, but in doing so, there existed more irregularity in the sleeve's appearance, therefore, no fixation was performed. The postoperative course was uneventful.

One year after primary SG, her DM was improved, being treated with monotherapy, and her nausea and vomiting never recurred.

\section{DISCUSSION}

GV can be present as an acute abdominal emergency or a chronic cause of abdominal pain. Borchardt triad for diagnosing acute GV consists of unproductive retching, epigastric pain, and inability to pass a nasogastric tube. ${ }^{8}$ Despite the fact that an upper endoscopy may show twisting of the gastric fold, it may not be prudent to rely on such a procedure if a gastric ischemia is suspected. ${ }^{1} \mathrm{GV}$ may be chronic if the rotation is minimal and there is no vascular compromise. Symptoms usually consist of mild intermittent upper abdominal pain, early satiety, bloating, and belching. Ischemia might be a complication, which can lead to gastric necrosis and, if untreated, shock and death. ${ }^{9}$ The stomach is strongly fixed proximally at the cardiac and distally by the retroperitoneal fixations of the duodenum. Supporting these 2 points, the gastrophrenic,

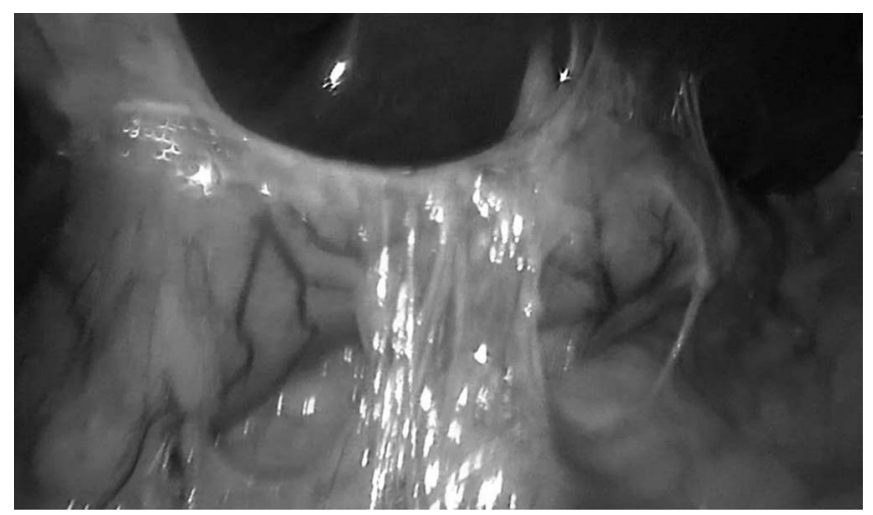

Figure 2. Gastric torsion secondary to adhesions between the sleeve's staple line and surrounding structures.

gastrosplenic, gastrocolic, and gastrohepatic ligaments hold the stomach in place ${ }^{10}$ in order to prevent GV. Even the agenesis of gastrocolic ligament only has been related with acute primarily GV with partial gastric necrosis. ${ }^{11}$

During the SG creation, the gastrophrenic, gastrocolic, gastrosplenic, and the posterior gastric attachments are divided, ${ }^{12,13}$ so the probability of twisting, turning, or folding is more likely to occur. In some cases during SG, the surgeon may observe a tendency of the new tubular stomach to form a coil shape that may cause obstructive symptoms. In order to prevent this coiling/twisting, some surgeons have recommended that fixation to the greater omentum to the stomach will keep it in the correct position. ${ }^{14}$

Because GV is well known in patients who have not had gastric surgery such as SG, we feel that it is best to describe those who have the similar findings of a twist, turn, or fold of the stomach after SG (or any other significant gastric surgery) as GT. This would avoid any confusion and make it clear that the patient's abnormality is likely due to surgical disruption of gastric attachments.

In our patients, the endoscopy was a very valuable tool for the establishment of GT (Fig. 1). The intraoperative, consistent finding was organoaxial torsion, secondary to the development of adhesions between the sleeve's staple line and the surrounding structures. This was due to lack of the normal gastric attachments, therefore, enabling the torsion (or twist, turn, fold) (Fig. 2).

The presence of the crural defects (hiatal hernia) on reoperation is likely caused by the obstructive nature of the GT, because we routinely explore every hiatus during primary SG to repair any defects and prevent postoperative reflux. 
Laparoscopic conversion to Roux-en-Y gastric bypass has been described successfully for acute gastric obstruction after SG. ${ }^{15}$ In the GV reported by Del Castillo et al, ${ }^{6}$ an antrectomy of the GS and then a gastroileal anastomosis was performed as an alternative option. Nassif et al 7 reported 1 case of twisting of the gastric tube (out of 55 patients who underwent $S G$ ) that responded satisfactorily to dilatation.

In our program, a total of 295 SGs were performed during the time the 3 patients described here had their sleeve gastrectomies. The mean time between SG and GT symptomatology was 111 days in our patient series. Treatment consisted of laparoscopic torsion reduction by lysis of adhesions in all patients, using the same trocar position as we did for primarily SG. Only in 1 patient (number 2) was a gastropexy to the retroperitoneum performed with absorbable 2-0 suture due to the presence of multiple anterior and posterior adhesions of the sleeve and its tendency to recoil.

\section{CONCLUSIONS}

Due to the fact that SG alters the normal stomach attachments, twisting, turning, or folding of the sleeve must be considered after SG in patients with any degree of obstruction symptoms, at any time of the postoperative course. We believe that when diagnosed, this entity should be termed GT to avoid confusion with the wellknown term GV in patients who have not had gastric surgery.

Endoscopy seems to be the most valuable tool for the diagnosis. Laparoscopic torsion reduction by lysis of adhesions was successful, with or without gastropexy, in our 3 cases.

\section{References:}

1. Jeyarajah DR, Harford WV Jr. Abdominal hernias and gastric volvulus. In: Feldman M, Friedman LS, Brandt, LJ, eds. Sleisenger and Fordtran's Gastrointestinal and Liver Disease. Vol. 1. 9th ed. New York, NY: Saunders; 2010:383-385.

2. Hutter MM, Schirmer BD, Jones DB, et al. First report from the American College of Surgeons Bariatric Surgery Center Network: laparoscopic sleeve gastrectomy has morbidity and effectiveness positioned between the band and the bypass. Ann Surg. 2011;245(3):410-422.
3. Lalor PF, Tucker ON, Szomstein S, Rosenthal RJ. Complications after laparoscopic sleeve gastrectomy. Surg Obes Relat Dis. 2008;4(1):33-38.

4. Frezza EE, Reddy S, Gee LL, Watchel MS. Complications after sleeve gastrectomy for morbid obesity. Obes Surg. 2009;19(6): $684-687$.

5. Buchwald H, Oien DM. Metabolic/bariatric surgery worldwide 2011. Obes Surg. 2013;23(4):427-436.

6. Del Castillo Déjardin D, Sabench Pereferrer F, Hernàndez Gonzàlez M, Blanco Blasco S, Cabrera Vilanova A. Gastric volvulus after sleeve gastrectomy for morbid obesity. Surgery. 2013; 153(3):431-433.

7. Nassif PA, Valadão JA, Malafaia O, Torres OJ, Garcia RF, Klostemann FC. Technical modification for sleeve gastrectomy. Arq Bras Cir Dig. 2013;26 Suppl 1:74-78.

8. Ajao OG. Gastric volvulus: a case report and review of the literature. J Natl Med Assoc. 1980;72(5):520-522.

9. Hess J, Lowell M. Esophagus, stomach, and duodenum. In: Marx J, Hockberger R, Walls R, eds. Rosen's Emergency Medicine: Concepts and Clinical Practice. Vol. 1. 8th ed. New York, NY: Saunders; 2014:1180-1181.

10. Smith RJ. Volvulus of the stomach. J Natl Med Assoc. 1983; 75(4):393-397.

11. Mazzei C, Palatucci V, Pollio A, della Corte M, Lombardi D. Partial gastric necrosis due to acute gastric volvulus secondary to agenesis of the gastrocolic ligament. Int J Colorectal Dis. 2013; 28(9):1315-1316

12. Akkary E, Duffy A, Bell R. Deciphering the sleeve: technique, indications, efficacy, and safety of the sleeve gastrectomy. Obes Surg. 2008;18(10):1323-1329.

13. Romero RJ, Kosanovic R, Rabaza JR, et al. Robotic sleeve gastrectomy: experience of 134 cases and comparison with a systematic review of the laparoscopic approach. Obes Surg. 2013;23(11):1743-1752.

14. Santoro S. Technical aspects in sleeve gastrectomy. Obes Surg. 2007;17(11):1534-1535.

15. Bellorin O, Lieb J, Szomstein S, Rosenthal RJ. Laparoscopic conversion of sleeve gastrectomy to Roux-en-Y gastric bypass for acute gastric outlet obstruction after laparoscopic sleeve gastrectomy for morbid obesity. Surg Obes Relat Dis. 2010;6(5): $566-568$. 